\title{
Locating the solar source of 13 April 2006 magnetic cloud
}

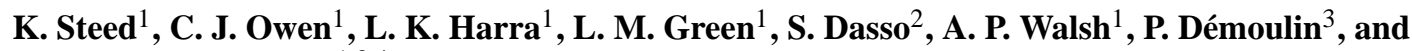 \\ L. van Driel-Gesztelyi ${ }^{1,3,4}$ \\ ${ }^{1}$ Mullard Space Science Laboratory, University College London, Holmbury St. Mary, Dorking, Surrey RH5 6NT, UK \\ ${ }^{2}$ Departmento de Física (FCEN-UBA) and Instituto de Astronomía y Física del Espacio, CONICET-UBA, CC. 67, Suc. 28, \\ 1428 Buenos Aires, Argentina \\ ${ }^{3}$ Observatoire de Paris, LESIA, UMR 8109 (CNRS), 92195 Meudon, France \\ ${ }^{4}$ Konkoly Observatory, Budapest, Pf. 67, 1525, Hungary
}

Received: 1 October 2007 - Revised: 31 January 2008 - Accepted: 26 May 2008 - Published: 15 October 2008

\begin{abstract}
Using Advanced Composition Explorer (ACE) in situ data we identify and describe an interplanetary magnetic cloud (MC) observed near Earth on 13 April 2006. We also use multi-instrument and multi-wavelength observations from the Solar and Heliospheric Observatory (SOHO), the Transition Region and Coronal Explorer (TRACE) and ground-based solar observatories to determine the solar source of this magnetic cloud.

A launch window for the MC between 9 and 11 April 2006 was estimated from the propagation time of the ejecta observed near Earth. A number of large active regions (ARs) were present on the Sun during this period, which were initially considered to be the most likely candidate source regions of the MC. However, it was determined that the solar source of the MC was a small, spotless active region observed in the Northern Hemisphere. Following an eruption from this region on 11 April 2006, the ACE spacecraft detected, $59 \mathrm{~h}$ later, the passage of the MC, preceded by the arrival of a weak, forward fast shock.

The link between the eruption in this active region and the interplanetary MC is supported by several pieces of evidence, including the location of the solar source near to the disk centre and to the east of the central meridian (in agreement with the spacecraft trajectory through the western leg of the magnetic cloud), the propagation time of the ejecta, the agreement between the amount of flux in the magnetic cloud and in the active region, and the agreement between the signs of helicity of the magnetic cloud and the active region (which differs from the sign of helicity of each of the other active regions on the Sun at this time). In addition, the active region is located on the boundary of a coronal hole, and a high speed solar wind stream originating from this region is observed near Earth shortly after the passage of the magnetic cloud.
\end{abstract}

\footnotetext{
Correspondence to: K. Steed

(ks3@mssl.ucl.ac.uk)
}

Keywords. Interplanetary physics (Interplanetary magnetic fields) - Solar physics, astrophysics, and astronomy (Flares and mass ejections)

\section{Introduction}

Magnetic clouds (MCs) are a subset of interplanetary coronal mass ejections (ICMEs), and are defined by three main characteristics: the magnetic field rotates smoothly through a large angle during an interval of the order of one day, the magnetic field strength is higher than that in the average solar wind, while the temperature is lower than that in the average solar wind (Burlaga et al., 1981; Burlaga, 1995). Richardson and Cane (1995) found that ICMEs typically have $T_{p} \leq T_{e x}$, where $T_{p}$ is the proton temperature and $T_{e x}$ is the "expected $T_{p}$ " determined from the empirical correlation between the velocity of the solar wind and $T_{p}$. Other large-scale solar wind structures, such as interplanetary sector boundaries, corotating interaction regions or post-shock ICME flows can exhibit any of the above features (Bothmer and Schwenn, 1992), but the combination of all three appears to be unique to magnetic clouds (e.g. Bothmer and Schwenn, 1998).

ICME identification remains a fairly ambiguous process since many of the signatures associated with ICMEs are not present for every ICME, and they often do not define precisely the same boundaries. This is expected since the various signatures arise from different physical phenomenon (e.g. Zwickl et al., 1983; Crooker et al., 1990; Richardson and Cane, 1995; Neugebauer and Goldstein, 1997; Mulligan et al., 1999; Richardson et al., 2003).

Structures on the Sun can be quantitatively linked to MCs at Earth for quiescent filament eruptions (e.g. Bothmer and Schwenn, 1998; Mulligan et al., 1998) and active regions (e.g. Qiu et al., 2007; Rodriguez et al., 2008), by considering the launch position on the Sun, the expected time delay

Published by Copernicus Publications on behalf of the European Geosciences Union. 


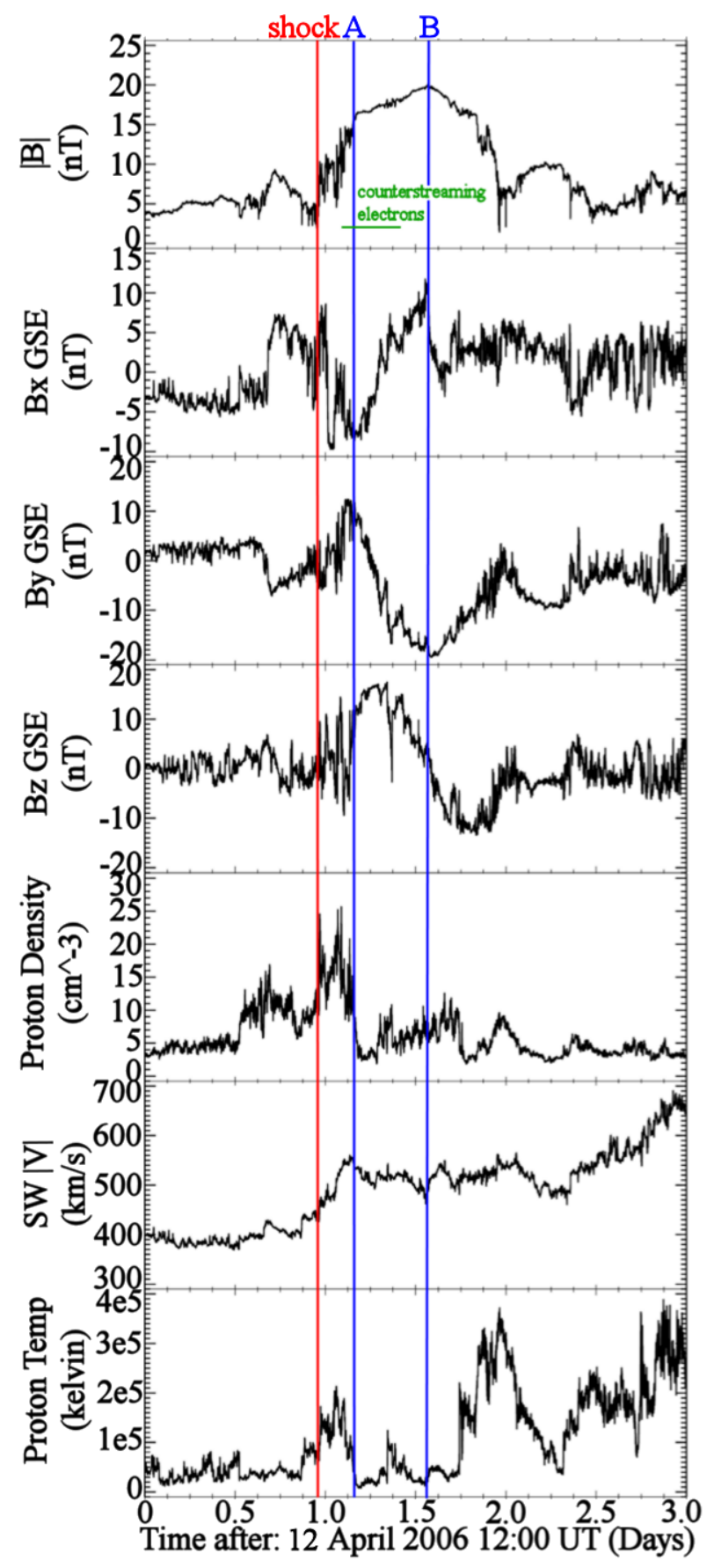

Fig. 1. Interplanetary magnetic field and plasma data obtained from the ACE spacecraft during the passage of the ICME ejecta. We plot the magnetic field intensity, $\boldsymbol{B}$, and its components in Geocentric Solar Ecliptic (GSE) co-ordinates, proton density, solar wind bulk speed and the proton temperature as a function of time. Vertical lines indicate the arrival of a shock (red) at $\sim 11: 00$ UT 13 April 2006 and the boundaries, A and B, (blue) of the magnetic cloud at $\sim$ 16:00 UT 13 April 2006 and 01:15 UT 14 April 2006, respectively. The duration over which counterstreaming, suprathermal electrons are observed is indicated by the horizontal green line between $\sim$ 14:30 UT and 22:00 UT 13 April 2006. and by comparing the orientation of the coronal and interplanetary magnetic field (Démoulin, 2008).

We first describe in Sects. 2 and 3 the components that comprise an ICME observed at $\sim 1$ AU on 13 April 2006, and the signatures from which this ejecta is identified as a magnetic cloud. We also define its boundaries from magnetic field and plasma parameters and describe the geometry of the MC. The time of eruption of the coronal mass ejection (CME) at the Sun is estimated and we then describe the process used to determine the solar source in Sect. 4, and discuss the elimination of several larger active regions as the solar source. In Sect. 5 we present the evidence that suggests the eruption originated from a small, Northern Hemisphere active region on 11 April 2006. Finally, in Sect. 6 we link the events observed near Earth and in the corona.

\section{Observational evidence of the ICME}

\subsection{Determining the boundaries of the ICME}

We analysed 16 second resolution data from the ACE/Magnetometer Instrument (MAG, Smith et al., 1998) and 64 second resolution data from ACE/Solar Wind, Electron, Proton and Alpha Monitor (SWEPAM, McComas et al., 1998), located in the vicinity of the Lagrangian point, L1, over the 3 day interval 12:00 UT on 12 April 2006 to 12:00 UT on 15 April 2006. Magnetic field intensity and its components in Geocentric Solar Ecliptic (GSE) coordinates, proton density, solar wind speed and proton temperature as a function of time are shown in Fig. 1 and were used to describe and characterise the magnetic cloud arriving at $\sim 1$ AU on 13 April 2006.

We observed the arrival of a weak, forward fast shock at ACE at 11:00 UT on 13 April 2006. This was identified in the data by simultaneous observations of discontinuities in the magnetic field intensity, proton density, solar wind bulk speed, and proton temperature, each of which were seen to increase at this time. This shock was produced because the speed of the leading edge boundary of the cloud relative to the solar wind was greater than the magneto-acoustic speed (Lepping et al., 1997). Following this, between $\sim 11: 00$ UT and $\sim 16: 00$ UT on 13 April 2006, the $B_{x}$,GSE, $B_{y, \text { GSE, and }}$ $B_{z, \mathrm{GSE}}$ components of the magnetic field become more variable, fluctuating rapidly. The proton density and temperature are also observed to peak during this interval, and the combination of these three signatures indicates the passage of the hot, dense pileup of the shocked "sheath" plasma, immediately preceding the arrival of the MC.

The identification of the MC and the locations of its boundaries were determined by considering a number of magnetic field and plasma signatures that are considered to be typical of magnetic flux ropes. A decrease in the variability of $\boldsymbol{B}$ was observed between $\sim 16: 00$ UT on 13 April 2006 and $\sim 01: 15$ UT on 14 April 2006, accompanied by 
an increase in $\boldsymbol{B}$ from $\sim 5 \mathrm{nT}$ to $\sim 20 \mathrm{nT}$. A smooth, coherent rotation of the $B_{x, \mathrm{GSE}}, B_{y, \mathrm{GSE}}$, and $B_{z, \mathrm{GSE}}$ components of the interplanetary magnetic field was also observed, with

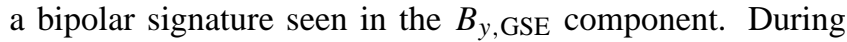
this interval, the proton temperature remains predominantly low, with values similar to pre-event levels of $<5 \times 10^{4} \mathrm{~K}$, even though the solar wind speed is higher than in the preevent region. Counterstreaming, suprathermal electrons were also observed during the passage of the ejecta, between $\sim$ 14:30 UT and 22:00 UT on 13 April 2006 .

\section{Determining the geometry of the MC}

Magnetic clouds are widely modelled as force-free, largescale, cylindrical magnetic flux ropes (e.g. Lepping et al., 1990; Lynch et al., 2003; Marubashi, 1997). The lines of force of the magnetic field given by the solution for a cylindrically symmetric force-free field with constant $\alpha$ are a family of helices with pitch increasing from the axis of the magnetic cloud (where the magnetic field is a straight line), to the boundary (where the lines of force are circles) (Burlaga, 1988).

The orientation of the axis of the observed MC was determined using a minimum variance analysis (MVA) of the magnetic observations (Sonnerup and Cahill, 1967). This method can be used to find the natural co-ordinate system of a flux rope. Figure 2 shows the magnetic field components in local magnetic coordinates obtained from the minimum variance analysis. The $B_{y}$,cloud panel shows a bipolar signature in the maximum variance direction and represents the rotation of the azimuthal field, whilst the axis of the cloud, the $B_{z, \text { cloud }}$ component, is in the intermediate direction.

Figure 3 shows the orientation of the magnetic cloud in the $y-z$ GSE plane. The MV analysis suggests that the axial magnetic field of the $\mathrm{MC}$ is mainly in the positive $z_{\mathrm{GSE}}$ direction and the maximum variance is mainly in the $y_{\mathrm{GSE}}$ direction, rotating from positive to negative. The MV analysis gives a ratio between the intermediate and the minimum eigen-values of $\sim 17$ (which implies that the minimum variance direction is well-defined (Siscoe and Suey, 1972)) and a low mean value and no large-scale trend for the $B_{x, \text { cloud }}$ series inside the $\mathrm{MC}$, which are arguments in favour of having a well-defined MC axis (Gulisano et al., 2007).

The MC axis orientation is described by $\theta$ and $\phi$, where $\theta$ is the angle between the ecliptic plane and the MC axis, and $\phi$ is the angle between $x_{\mathrm{GSE}}$ and the projection of the MC axis on the ecliptic plane (measured positive when anticlockwise). We find that $\theta \sim 68^{\circ}$ and $\phi \sim 294^{\circ}$, so the projection of the axis of the cloud on the ecliptic plane points predominantly towards the solar west (negative $y_{\text {GSE }}$ direction) with a positive $x_{\mathrm{GSE}}$ component. The field rotation implies that the $\mathrm{MC}$ is left-handed and this means that the sign of the helicity of the $\mathrm{MC}$ is negative.
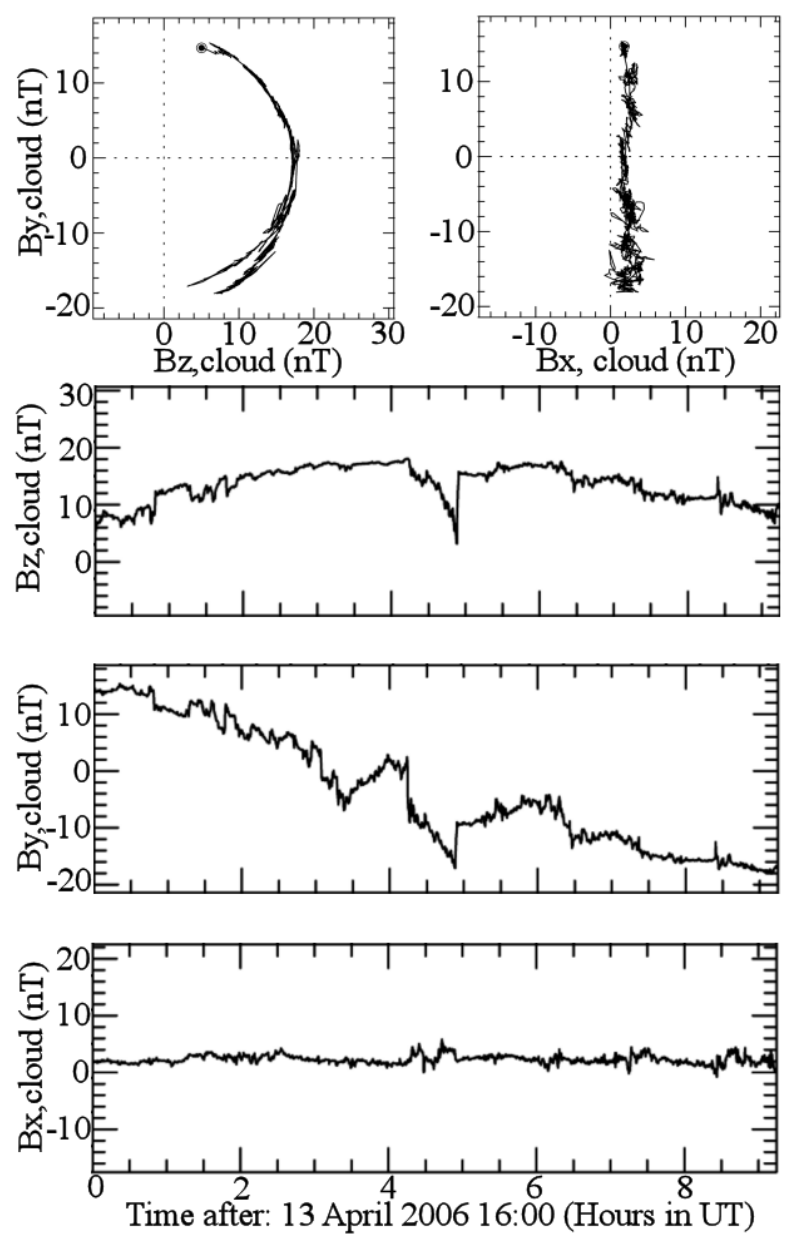

Fig. 2. Magnetic field components in local magnetic coordinates from the minimum variance analysis performed over the duration of the magnetic cloud (16:00 UT 13 April 2006-01:15 UT 14 April 2006). The two upper panels show the hodograms for the MC. The upper left panel shows the coherent rotation, while the upper right panel shows the noise in the $x$, cloud direction. The lower three panels show the evolution of the magnetic field components (with the orientation of the cloud given by the minimum variance method, see Sect. 3).

The spacecraft impact parameter (the minimum distance from the spacecraft trajectory to the axis of the cloud), $p$, is estimated using the method described by Gulisano et al. (2007), which is valid for the force free Lundquist (1950) model. The deviation from zero of the mean value of the $B_{x, \text { cloud }}$ component of the magnetic field (in the direction of minimum variance) can be used to obtain an estimation of $p$, as $p \approx R \sqrt{\left.<B_{x, \text { cloud }}>/ 1.6 \boldsymbol{B}_{\mathbf{0}}\right)}$, where $R$ is the radius of the $\mathrm{MC}$ and $\boldsymbol{B}_{\mathbf{0}}$ is the central field strength.

$B_{x, \text { cloud }}$ is expected to be zero when the spacecraft passes through the centre of the MC (when $p=0$ ). For this MC, the

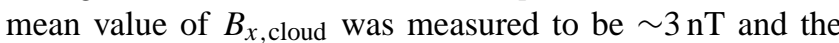
magnetic field at the cloud axis, $\boldsymbol{B}_{\mathbf{0}}$, can be estimated as the 


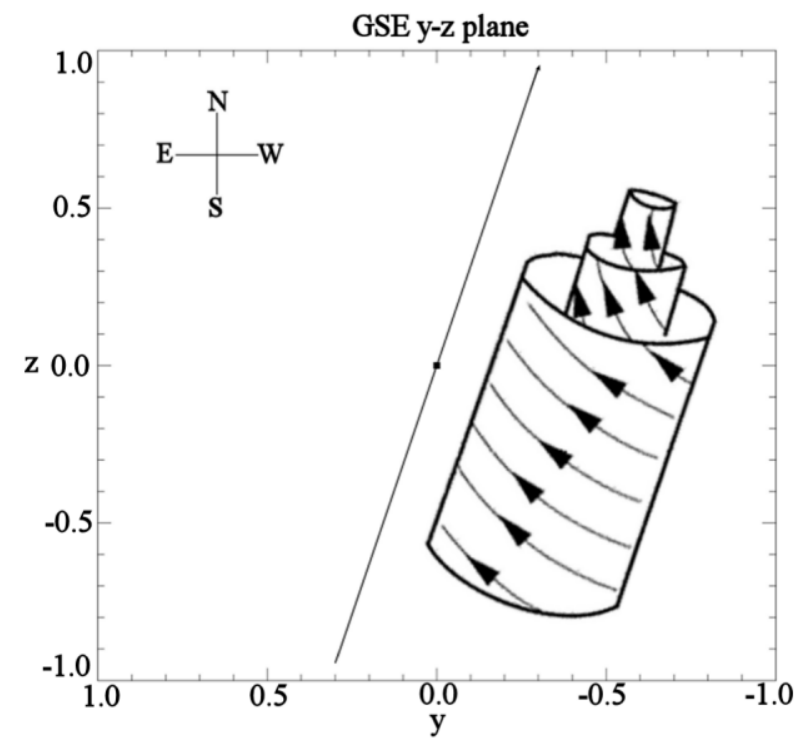

Fig. 3. The orientation of the magnetic cloud obtained from performing a minimum variance analysis over the cloud duration is illustrated in the $y-z$ GSE plane. The axial field is mainly in the positive $z_{\mathrm{GSE}}$ (northward) direction, whilst the rotation of the MC is mainly in the $y_{\mathrm{GSE}}$ direction, rotating positive to negative (east to west). This indicates that the MC is left-handed and the sign of helicity of the MC is negative. (Adapted from Bothmer and Schwenn, 1998).

maximum observed field because at this position the magnetic field is practically axial ( $B_{z, \text { cloud }}$ is the largest field component by a factor $\approx 6$ ); thus $\boldsymbol{B}_{0} \sim 18 \mathrm{nT}$. A value for $p / R$ of $\sim 30 \%$ was obtained (with the true central field strength $\boldsymbol{B}_{\mathbf{0}}$, or an estimate with fitting a model to the data, this estimate of $p$ would only be slightly lower).

From the measured velocity, the spacecraft trajectory through the cloud and the time period over which ACE observes the cloud, we estimate a radius, $R \sim 5.7 \times 10^{-2} \mathrm{AU}$, which implies a small magnetic cloud $(\sim 3$ times smaller than average (Lepping et al., 1990)).

\subsection{Magnetic flux of the MC}

From the value obtained for the radius of the cloud and an estimate of the magnetic field at the cloud axis, $\boldsymbol{B}_{\mathbf{0}}$, the classical, linear force-free model of Lundquist (1950), $\nabla \times \boldsymbol{B}=\alpha \boldsymbol{B}$, with constant $\alpha$ (e.g. Burlaga et al., 1981; Burlaga, 1988; Lepping et al., 1990) can be used to estimate the flux within the magnetic cloud. The axial flux, $F_{z}$, is $\sim 2.0 \times 10^{20} \mathrm{Mx}$ and the azimuthal flux, $F_{\phi}$ per unit length, is $\sim 1.1 \times 10^{21} \mathrm{Mx} / \mathrm{AU}$. The azimuthal flux within the MC is greater than the axial flux, as is expected for a highly twisted flux rope.

\subsection{Effects of the boundary selection}

The rear boundary of this magnetic cloud is not clearly defined; however from using the expected conservation of the magnetic flux in closed structures, such as flux ropes (Dasso et al., 2006), and fixing the start time as 16:00 UT on 13 April, we can select the rear boundary to be near 01:15 UT, where there is also a discontinuity in $B_{x, \mathrm{GSE}}$, as is expected to be observed at current sheets that separate different magnetic connectivities (Dasso et al., 2006).

A number of locations for the rear boundary were explored before the boundary at $\sim 01: 15$ UT on 14 April 2006 was selected. For each of the preliminarily identified boundaries we performed an MV analysis over the MC duration, and this indicated that changes in the rear boundary location between $\sim 01: 15$ UT and $\sim 11: 00$ UT 14 April 2006 do not result in major changes in the orientation of the $\mathrm{MC}$ axis.

\section{Locating the solar source of the ejecta}

\subsection{Estimating the launch window of the $\mathrm{MC}$}

The average speed of the ejecta near Earth was measured to be $520 \mathrm{~km} \mathrm{~s}^{-1}$. Assuming that this remained relatively constant as the MC propagated away from the Sun, we might expect a propagation time of $80 \mathrm{~h}$ from the corona to $1 \mathrm{AU}$, calculated from the arrival time of the centre of the MC. Selecting the time of arrival of the centre of the cloud, rather than that of the shock front, reduces the likelihood of mixing up the expansion of the structure and its global motion in the solar wind. This suggests that the CME is likely to have originated at the Sun at $\sim 12: 00$ UT on 10 April 2006. However, since the ICME was travelling significantly faster than the solar wind, the ejecta is unlikely to have been propagating at a constant speed and was most probably decelerated as it propagated into interplanetary space (Reiner et al., 2007). A suitable launch window for the magnetic cloud was considered to be between 9 April 2006 and 11 April 2006.

An initial survey of the Sun during this launch window revealed none of the more obvious CME signatures that might be expected. No large, eruptive flares, and no large propagating dimmings or deep dimmings were observed, making the source region of the MC more difficult to determine.

\subsection{Survey of possible source regions}

There were four known active regions on the Sun at 12:00 UT on 10 April 2006. AR10871 was located on the east limb, AR10866 and AR10867 were located on the west limb, and AR10869 was located slightly south-west of the centre of the disk. There was also a region of emerging flux east of disk centre, which later developed into AR10870. A small active region north-east of disk centre emerged on 10 April 2006, but did not become sufficiently large to develop sunspots or be allocated a NOAA active region number. The 
active regions on the limbs were much larger than those on the disk, but any CMEs erupting from these regions were considered unlikely to be directed towards the Earth, since a CME is expected to propagate away from the Sun approximately radially (Hundhausen, 1994; St. Cyr, 1999, 2000).

The SOHO/Extreme-Ultraviolet Imaging Telescope (EIT, Delaboudinière et al., 1995), which observes the full Sun regularly with four different filters and with a pixel size of 2.6 arcsec, was initially used to look for any eruptive activity in the active regions present on and around 10 April 2006. At 05:47 UT on 10 April 2006, an EUV brightening was observed at $195 \AA$ corresponding to a GOES B4.4 class X-ray flare in active region 10869 with an onset time of 05:42 UT (peaking at 05:48 UT), indicating possible reconnection following an eruption in this region. The EIT observations of the ejecta, combined with the location of the active region near to the disk centre and the good agreement of the time of the eruption with the propagation time estimated for the ejecta to travel from the corona to $\sim 1 \mathrm{AU}$, suggests that this CME might be the solar source of the magnetic cloud observed near Earth on 13 April 2006. However, it is necessary to check other association criteria and analyse any other possibility of eruption from other regions before concluding.

\subsubsection{Determining the magnetic helicity signs of possible source regions}

Magnetic helicity is an approximately conserved quantity in resistive MHD with low resistivity (Berger, 1984) and provides a quantitative link between eruptions from the corona and corresponding CMEs observed in situ at $1 \mathrm{AU}$. Thus, the erupting structure in the solar corona is expected to have the same helicity sign as the ICME (Bothmer and Rust, 1997). For a magnetic flux rope, the helicity is given by $H=\Phi^{2}\left(T_{w}+W_{r}\right)$, where $\Phi$ is the axial magnetic flux within the rope, $T_{w}$ is the twist or number of windings of the field lines around the axis, and $W_{r}$ is the writhe; which is a measure of the distortion of the flux rope axis. For a cylindrical flux rope with little or no writhe, the sign of helicity of the rope equals the sign of twist. Positive helicity corresponds to positive (right-handed) twist and negative helicity to negative (left-handed) twist.

There are several ways to determine the sign of helicity in the coronal field (Démoulin, 2008), however in the present case, there is only one way to determine the sign of helicity from the available data. When the apex of a flux rope emerges across the photosphere, the presence of twist produces a contribution of the azimuthal field projected onto the line-of-sight component. This is observed in longitudinal magnetogram data as two elongated "tongues" of opposite polarities in the active region field (López Fuentes et al., 2000). For a positive twist, the leading polarity appears elongated and extends southward of the following polarity. The situation is the mirror image for a negative sign of twist.
A positive twist is expected in Southern Hemisphere ARs according to the hemispheric chirality rules (Pevtsov and Balasubramaniam, 2003). Further investigation of AR10869, located in the Southern solar Hemisphere, indicated that this is likely to be the case for this active region. Since the sign of helicity of this AR does not seem to be in agreement with the negative sign of helicity of the magnetic cloud, AR10869 is unlikely to be the solar source of the MC.

An inspection of the helicity signs of all of the remaining active regions on the Sun at this time by considering magnetic "tongues" when the apex of the flux rope was crossing the photosphere, indicated that it is likely that all except one; the small, Northern Hemisphere active region, had a positive sign of helicity. Assuming conservation of magnetic helicity, this allowed us to rule out each of the regions with an opposite helicity sign to that of the MC.

\subsubsection{Disappearing filaments}

Filaments, or eruptive prominences frequently accompany CMEs (e.g. Hundhausen, 1993; Hanaoka et al., 1994; Gopalswamy et al., 1996). When a filament begins to rise during the eruption of a CME, the $\mathrm{H} \alpha$ emission is Doppler shifted and may no longer be visible in narrow band images. These are known as "disappearing filaments" (Pick et al., 2006). They represent a good proxy for the configuration of the coronal magnetic field: they over-lie polarity inversion lines and the surrounding coronal magnetic fields are highly sheared. CMEs accompanied by a disappearing filament are useful in order to try and predict their magnetic topology (Bothmer and Schwenn, 1994, 1998; Mulligan et al., 1998).

$\mathrm{H} \alpha$ observations, obtained from a number of solar observatories, show several filaments present on the disk during the launch window of the magnetic cloud. Some small filaments can be identified in the active regions, whilst there are also some quiet-Sun filaments present in both the Northern and Southern Hemispheres. The evolution of the only visible quiet-Sun filament located in the Northern Hemisphere, which was expected to have negative helicity, was studied to determine if the magnetic cloud could have originated from a quiet-Sun source, but this filament is not seen to erupt between 9 and 11 April 2006.

The spatial resolution of the $\mathrm{H} \alpha$ data was not sufficient to resolve any filaments or filament eruptions in the Northern Hemisphere active region.

\subsubsection{Coronagraph observations}

A very faint, partial halo CME was observed at $~ 06: 00$ UT on 10 April 2006 by the SOHO/Large Angle Spectroscopic Coronagraph (LASCO, Brueckner et al., 1995), extending towards the south west of the Sun. This is the only partial halo CME recorded in the LASCO CME catalog during the launch window of the MC, and this was verified by 

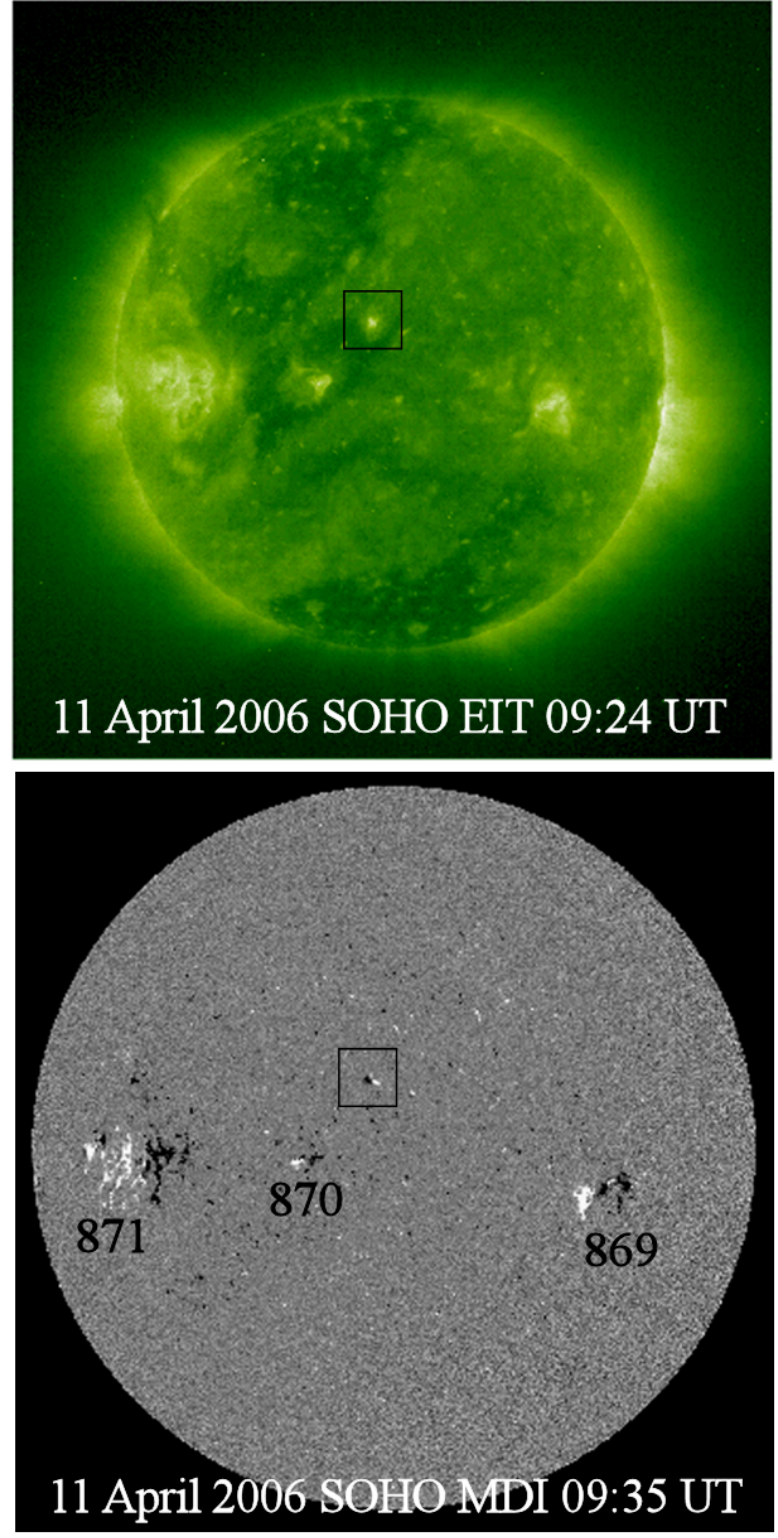

Fig. 4. The locations of all of the active regions on the visible disk of the Sun at the time at which the CME associated with the magnetic cloud erupted. At 09:24 UT on 11 April 2006, there are four active regions on the Sun observed by SOHO/EIT at $195 \AA$ and observed a short time later by SOHO/MDI. The eruption originates from a small, spotless active region located slightly north east of disk centre (shown within the boxes). AR10869 is located towards the west limb, and AR10870 and AR10871 are both located east of the central meridian. All of the active regions that have been allocated NOAA active region numbers are situated in the Southern solar Hemisphere.

a visual inspection of the LASCO data. The first observation of this CME was by the C2 coronagraph at 06:06 UT, and at 11:18 UT the CME became visible in the $\mathrm{C} 3$ corona- graph. The linear speed and the estimated onset time of this CME recorded in the LASCO CME catalog are $183 \mathrm{~km} \mathrm{~s}^{-1}$ and $\sim 04: 00 \mathrm{UT}$, respectively. A lack of eruption signatures on the solar disk corresponding to this CME have led us to conclude that this was a back-sided event.

\section{Analysis of the Northern Hemisphere solar source}

Using the SOHO/Michelson Doppler Imager (MDI, Scherrer et al., 1995), observations of the small Northern Hemisphere active region (identified in Fig. 4) can be made just as the apex of the $\Omega$ loop is emerging. It is seen in magnetograms of this AR from SOHO/MDI in Fig. 5 that the leading (positive) polarity extends northward of the following (negative) polarity during the early emergence phase. This indicates that the emerging flux rope contained negative helicity. The schematic included in Fig. 5 more clearly illustrates the relative positions of two elongated "tongues" of opposite polarities when a region has a negative sign of helicity.

At 09:24 UT on 11 April 2006, a cusp was observed in this AR by SOHO/EIT at $195 \AA$ and the formation of posteruption loops was observed by the Transition Region and Coronal Explorer (TRACE, Handy et al., 1999) at $171 \AA$. Figure 6 shows the coronal evolution of this active region in EUV with SOHO/EIT and TRACE. Highlighted are cuspshaped coronal loops that are thought to be produced as a result of the reconnection of field lines previously opened by an eruption, so provide a signature of eruption (Sturrock and Smith, 1968).

This active region is situated on the boundary of a coronal hole. Whereas ARs contain mainly closed magnetic field lines, coronal holes are regions of low emission dominated by "open" magnetic field lines (Kopp and Holzer, 1976). Despite the small size of this active region, its location means that it might be expected to erupt more readily than if it were situated in the quiet Sun. Reconnection between the active region field and the coronal hole field leads to closed AR fields being opened. This removes field overlying the core of the AR and leads to the rapid decrease of the field strength with respect to height, and increases the ease of the escape of the ejecta (Baker et al., 2007; Török and Kliem, 2007).

Liu (2007) showed observationally that CME source regions in coronal holes produce fast ejecta (with speeds of up to $2000 \mathrm{~km} \mathrm{~s}^{-1}$ ). This suggests that the velocity of this eruption may be faster than might be expected for a region of this size. Observations of the MC near Earth indicate that it was propagating at a velocity of $520 \mathrm{~km} \mathrm{~s}^{-1}$. However for this CME to travel $1 \mathrm{AU}$ in only $59 \mathrm{~h}$, it must have had a substantially faster initial velocity, which decreased as it propagated away from the Sun. We estimate that the mean velocity of this ejecta, during its transit from the Sun to $1 \mathrm{AU}$, must have been nearer to $710 \mathrm{~km} \mathrm{~s}^{-1}$.

SOHO/MDI returns the line of sight (longitudinal) flux density, averaged over the pixel field of view. Multiplying 

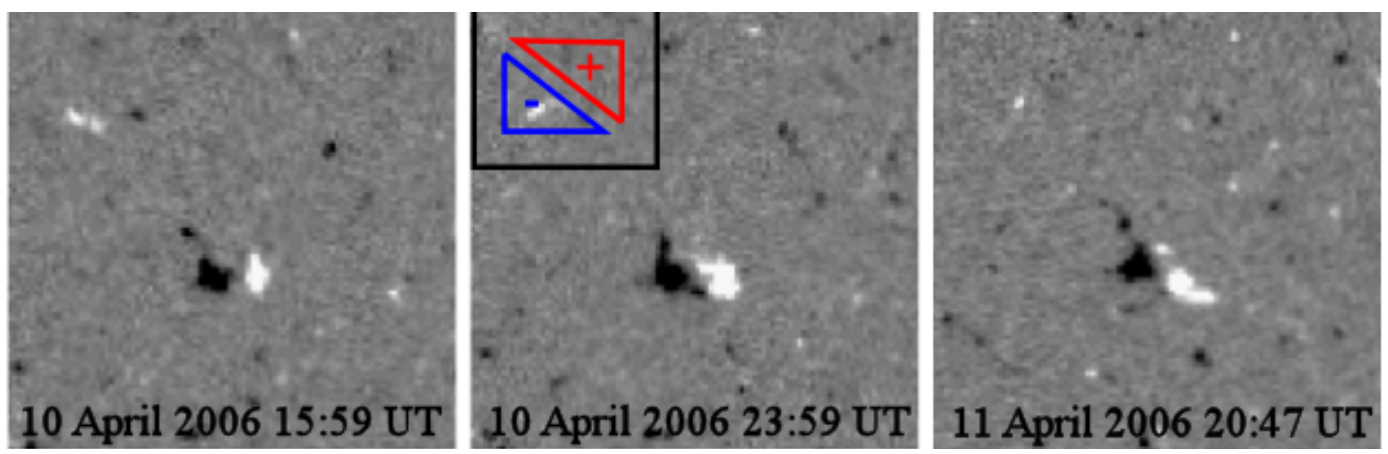

Fig. 5. Longitudinal magnetogram observations from SOHO/MDI of flux emerging in the small, Northern Hemisphere active region. White corresponds to positive polarity magnetic field, whilst black corresponds to negative polarity magnetic field. The first magnetogram (left) shows magnetic flux in the active region soon after it begins to emerge. In the second magnetogram (centre) the leading (positive) polarity field extends to the north of the following (negative) polarity field. Included on this magnetogram is a schematic illustrating the relative positions of two elongated "tongues" of opposite polarities when the emerging active region has a negative sign of helicity, as in the case of this active region. The last magnetogram (right) shows the well separated footpoints of the developed active region.

the flux density by a given area then gives the total flux contained within this area. To measure the magnetic flux within an active region a polygonal contour, defined by visual inspection, is fitted around the region and the flux is summed within it. The MDI instrument requires some corrections and for this we follow the method of Berger and Lites (2003). In our data, the flux values must be corrected by a factor of 1.45 which gives the magnetic flux of the active region (average between values obtained for the positive and negative fluxes) to be $7( \pm 0.4) \times 10^{20} \mathrm{Mx}$.

\section{Linking the coronal eruption to the interplanetary $\mathrm{MC}$}

In Sects. 2 and 3 we described an interplanetary MC observed near Earth on 13 April 2006. This MC and the coronal eruption described in Sect. 5, have properties that indicate their probable association, as follows.

- The eruption occurs close to disk centre, and since CMEs predominantly propagate away from the Sun in a radial direction, this implies that the event could also have been observed by ACE.

- Two and a half days travel time means that this eruption occurs within the expected launch window of the CME, given that this CME must have erupted from the Sun with a much higher initial velocity than the velocity of the MC measured near Earth, due to its location on the boundary of a coronal hole.

- The magnetic helicity sign of the MC and that of the small, Northern Hemisphere active region are in agreement, providing evidence of the same region ICME connection.
- From the in situ data, it is predicted that the ACE spacecraft crossed the western leg of the MC (since $\phi \sim 294^{\circ}$, the $\mathrm{MC}$ axis is in the quadrant defined by $x_{\mathrm{GSE}}>0$ and $\left.y_{\mathrm{GSE}}<0\right)$. This is in agreement with the eastern position of the source region with respect to the central meridian.

- The magnitude of the flux within the magnetic cloud does not exceed the calculated flux within the active region.

Moreover, the location of the source region near to the central meridian at the time of the eruption, together with its close proximity to a coronal hole that is situated to the east of this AR explains the presence of a high speed solar wind stream following the magnetic cloud. Prior to the arrival of the MC, the solar wind speed is representative of the average speed of the slow solar wind propagating at $\sim 400 \mathrm{~km} \mathrm{~s}^{-1}$. The propagation speed of the magnetic cloud is faster than this solar wind flow, and this consequently results in the formation of a forward fast shock. Shortly after the time at which the CME erupts from the small, Northern Hemisphere AR, the coronal hole passes the central meridian of the Sun and a high speed solar wind stream originating from this region is observed near Earth, with speeds reaching up to $\sim 700 \mathrm{~km} \mathrm{~s}^{-1}$ following the passage of the MC.

\section{Discussion and conclusions}

The ICME observed near Earth on 13 April 2006 was identified as a magnetic cloud. The magnetic field rotated smoothly, the magnetic field strength was elevated, and the proton temperature was low for a duration of $\sim 9 \mathrm{~h}$. Analysis of the geometry of the cloud implied that it was orientated approximately in the direction east-north-west, and that it had left-handed (negative) twist. 

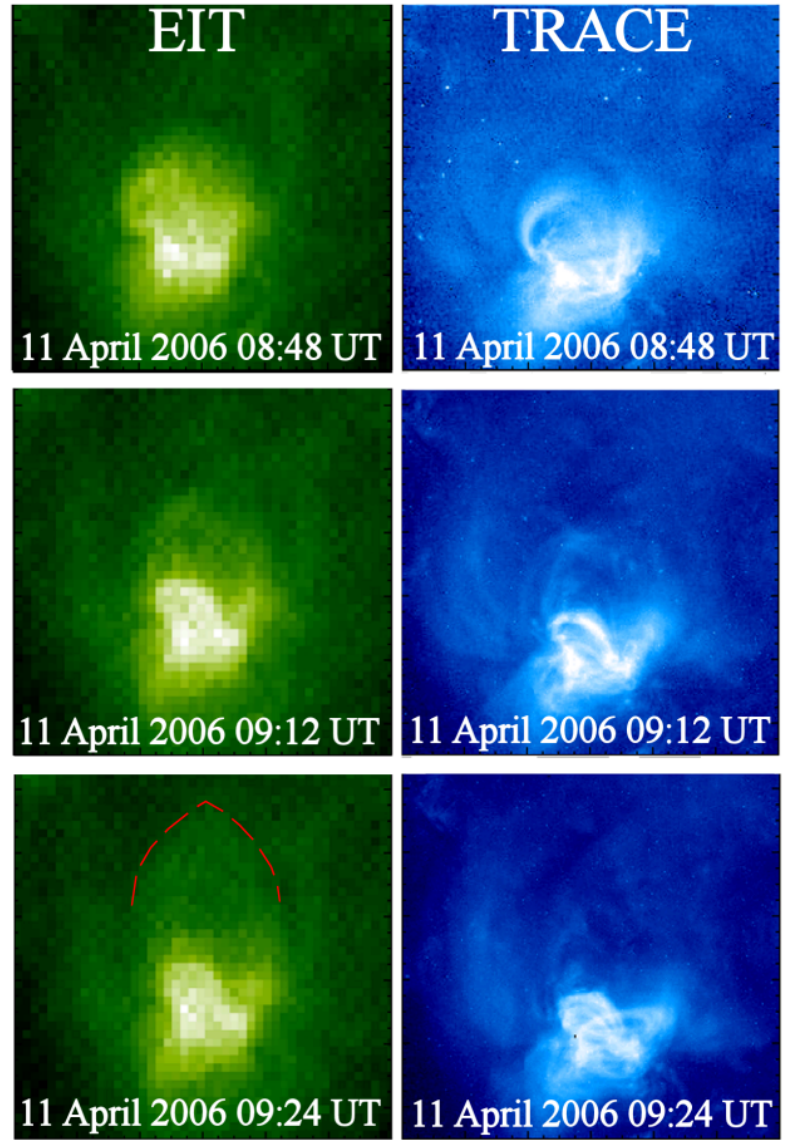

Fig. 6. Coronal evolution of the small, Northern Hemisphere active region prior to and following the eruption that occured in this region at $\sim 09: 24$ UT on 11 April 2006. The left column shows data from SOHO/EIT at $195 \AA$. A cusp-shaped coronal loop is oberved at 09:24 UT (red dashed line) providing evidence of an eruption in this region. The right column shows data from TRACE at $171 \AA$, in which post-eruption loops are observed coinciding with the time of the eruption estimated from the SOHO/EIT observations. The field of view of these images is $120 \times 120 \operatorname{arcsec}(2.62 \operatorname{arcsec}$ per pixel).

There was eruptive activity in a small, Northern Hemisphere active region for which the timing was in agreement with the estimated propagation time required for the $\mathrm{CME}$ to travel from the corona to $1 \mathrm{AU}$. Evidence for this eruption included a cusp and post-eruption loops identified in EUV observations. At this time, this appears to be the only active region on the visible disk with a negative sign of helicity, in agreement with the sign of helicity of the cloud.

Combining observations of the same event of both the Sun and in situ near Earth helps us to investigate the magnetic topology of the erupting structure directly, and understanding the magnetic topology of the structure is crucial to the understanding of how and why an eruption occured. There are several possible eruption scenarios for this event:
1. Simple expansion of coronal loops or pre-existing flux rope. In this scenario the axial flux of the magnetic cloud should match the longitudinal flux in the dimmings (which is thought to reveal the "feet" of the expanding and erupting magnetic structure). However, the eruption studied in this event showed no dimming signature. In this case, the longitudinal flux contained within the active region sets an upper limit for what is expected to be detected in the MC. In our case this holds, as we find that the active region flux is a factor of 3.5 larger than the axial flux of the MC.

2. Flux rope formation during the eruption. In this scenario the flux rope was not present before the eruption, but instead was formed through successive reconnection as the eruption proceeded. This means that the azimuthal field of the MC comes purely from reconnection which not only produces the ejected flux rope, but also produces the flare loops below. As a result of this Qiu et al. (2007) propose that in this case the azimuthal flux is approximately equal to the reconnection flux observed in the flare ribbons. In our case no flare ribbons were produced so we cannot further investigate this scenario.

3. Eruption of a flux rope with reconnection proceeding underneath. In this scenario (also known as the CSHKP scenario (Carmichael, 1964; Sturrock, 1966; Hirayama, 1974; Kopp and Pneuman, 1976), as the flux rope rises reconnection proceeds below it. This reconnected flux closes down to form flare loops and up to add azimuthal flux to the pre-existing flux rope. In this case, Qiu et al. (2007) propose that the azimuthal flux is greater than the reconnected flux. Again, the absence of flare ribbons means that this scenario cannot be tested.

We do not have sufficient observational evidence to determine with any certainty which of these scenarios is most likely to have occured for this event, although we do observe a cusp which indicates that reconnection does take place, even though it is not energetic enough to produce a solar flare. The presence of reconnection in the AR combined with the magnetic fluxes measured in the AR and in the MC favours the second or third scenarios.

Mandrini et al. (2005) studied an eruption from an X-ray bright point situated within a coronal hole; which formed the smallest magnetic cloud observed to that point in time. Although there are similarities between our event and that studied by Mandrini et al., there are also some interesting differences. The eruption in Mandrini et al. (2005) produces a transition from sigmoid to cusp in the structure of the X-ray bright point, and twin dimmings. These can be used to investigate the erupting field, and Mandrini et al. propose that the longitudinal flux in the dimmings $(60 \%$ of the $\mathrm{X}$-ray bright point's flux) is well correlated with the azimuthal flux in the magnetic cloud. In our case, the observational signatures are far more subtle and we are unable to investigate this aspect. 
Also, there is a factor of 2 difference between the source region flux in these two case studies, yet a factor of 10 difference in the axial field and the azimuthal field per unit length between the studied magnetic clouds, with the MC cloud in this work being the larger in all cases.

This event highlights the complexities associated with locating the solar source of an ICME observed near Earth, and serves to emphasise that it is the combination of a number of physical characteristics and signatures that is important for successfully tying together the Earth-end and the Sun-end of an event.

Acknowledgements. The authors thank the SOHO/MDI and EIT consortia for their data, and the NASA's Space Physics Data Facility (SPDF) for the interplanetary observations. The LASCO instrument was constructed by a consortium of the Naval Research Laboratory, University of Birmingham (United Kingdom), the Max-Planck-Institut für Aeronomie (Germany), and the Laboratoire d'Astronomie Spatiale (France). We acknowledge the use of solar wind data from the on-line CDAW Web site of NASA/GSFC. The ACE magnetic field and plasma data are courtesy of N. Ness and D. J. McComas respectively. The CME catalog is generated and maintained at the CDAW data centre by NASA and The Catholic University of America in cooperation with the Naval Research Laboratory. SOHO is a project of international collaboration between ESA and NASA. K. Steed thanks STFC for support via Ph.D. studentship. S. Dasso is a member of the Carrera del Investigador Cientifico del CONICET. S. Dasso acknowledges partial support from grants PIP6220 (CONICET) and PICT 05-33370 (FONCyT).

Topical Editor R. Forsyth thanks two anonymous referees for their help in evaluating this paper.

\section{References}

Baker, D., van Driel-Gesztelyi, L., and Attrill, G. D. R.: Evidence for interchange reconnection between a coronal hole and an adjacent emerging flux region, Astronomische Nachrichten, 328, 773-776, 2007.

Berger, M. A.: Rigorous new limits on magnetic helicity dissipation in the solar corona, Geophys. Astrophys. Fluid Dynam., 30, 79104, 1984.

Berger, T. E. and Lites, B. W.: Weak-field magnetogram calibration using Advanced Stokes Polarimeter flux density maps - II. SOHO/MDI full-disk mode calibration, Sol. Phys., 213, 213229, 2003.

Bothmer, V. and Schwenn, R.: Magnetic structures at sector boundaries in the inner heliosphere, Solar Wind Seven, Proceedings of the 3rd COSPAR colloquium, Goslar, Germany, 151-154, 1992.

Bothmer, V. and Schwenn, R.: Eruptive prominences as sources of magnetic clouds in the solar wind, Space Sci. Rev., 70, 215-220, 1994.

Bothmer, V. and Rust, D. M.: The field configuration of magnetic clouds and the solar cycle, Geophys. Mono., 99, 139-146, 1997.

Bothmer, V. and Schwenn, R.: The structure and origin of magnetic clouds in the solar wind, Ann. Geophys., 16, 1-24, 1998, http://www.ann-geophys.net/16/1/1998/.
Brueckner, G. E., Howard, R. A., Koomen, M. J., et al.: The Large Angle Spectroscopic Coronagraph (LASCO), Sol. Phys., 162, 357-402, 1995.

Burlaga, L., Sittler, E., Mariani, F., and Schwenn, R.: Magnetic loop behind an interplanetary shock: Voyager, Helios and IMP 8 observations, J. Geophys. Res., 86, 6673-6684, 1981.

Burlaga, L. F.: Magnetic clouds and force-free fields with constant alpha, J. Geophys. Res., 93, 7217-7224, 1988.

Burlaga, L. F.: Interplanetary Magnetohydrodynamics, Oxford Univ. Press, New York, 89-114, 1995.

Carmichael, H.: A process for flares, in: AAS-NASA Symposium on Solar Flares, edited by: Hess, W. N. (NASA SP-50), 451, 1964.

Crooker, N. U., Gosling, J. T., Smith, E. J., and Russell, C. T.: A bubblelike coronal mass ejection flux rope in the solar wind, in: Physics of Magnetic Flux Ropes, edited by: Russell, C. T., Priest, E. R., and Lee, L. C., Geophys. Monogr. Ser. Vol. 58, 365, AGU., Washington D.C., 1990.

McComas, D. J., Bame, S. J., Barker, P., Feldman, W. C., Phillips, J. L., Riley, P., and Grifee, J. W.: Solar Wind Electron Proton Alpha Monitor (SWEPAM) for the Advanced Composition Explorer, Space Sci. Rev., 86, 563-612, 1995.

Dasso, S., Mandrini, C. H., Démoulin, P., and Luoni, M. L.: A new model-independent method to compute magnetic helicity in magnetic clouds, Astron. Astrophys., 455, 349-359, 2006.

Démoulin, P.: A review of the quantitative links between CMEs and magnetic clouds, Ann. Geophys., 26, 3113-3125, 2008, http://www.ann-geophys.net/26/3113/2008/.

Delaboudinière, J.-P., Artzner, G. E., Brunaud, J., et al.: EIT: Extreme-Ultraviolet Imaging Telescope for the SOHO Mission, Sol. Phys., 162, 291-312, 1995.

Gopalswamy, N., Kundu, M. R., Hanaoka, Y., Enome, S., Lemen, J. R., and Akioka, M.: Yohkoh/SXT observations of a coronal mass ejection near the solar surface, New Astron., 1, 207-213, 1996.

Gulisano, A. M., Dasso, S., Mandrini, C. H., and Démoulin, P.: Estimation of the bias of the minimum variance technique in the determination of magnetic clouds global quantities and orientation, Adv. Space Res., 40, 1881-1890, 2007.

Hanaoka, Y., Kurokawa, H., Enome, S., et al.: Simultaneous observations of a prominence eruption followed by a coronal arcade formation in radio, soft x-rays and $\mathrm{H}($ alpha), Publ. Astron. Soc. Jpn., 46, 205-216, 1994.

Handy, B. N., Acton, L. W., Kankelborg, C. C., Wolfson, C. J., Akin, C. J., et al.: The transition region and coronal explorer, Sol. Phys., 187, 229-260, 1999.

Harrison, R. A., Bryans, P., Simnett, G. M., Lyons, M.: Coronal dimming and the coronal mass ejection onset, A. \& A., 400, 1071-1083, 2003.

Hirayama, T.: Theoretical model of flares and prominences, Sol. Phys., 34, 323-338, 1974.

Hundhausen, A. J.: Sizes and locations of coronal mass ejectionsSMM observations from 1980 and 1984-1989, J. Geophys. Res., 98, 13 177-13 200, 1993.

Hundhausen, A. J., Burkepile, J. T., and St. Cyr, O. C.: Speeds of coronal mass ejections: SMM observations from 1980 and $1984-$ 1989, J. Geophys. Res., 99, 6543-6552, 1994.

Kopp, R. A. and Holzer, T. E.: Dynamics of coronal hole regions, Sol. Phys., 49, 43-56, 1976. 
Kopp, R. A. and Pneuman, G. W.: Magnetic reconnection in the corona and loop prominence phenomenon, Sol. Phys., 50, 8598, 1976.

Lepping, R. P., Jones, J. A., and Burlaga, L. F.: Magnetic field structure of interplanetary magnetic clouds at 1 AU, J. Geophys. Res., 95, 11957-11965, 1990.

Lepping, R. P., Burlaga, L. F., Szabo, A., et al.: The WIND magnetic cloud and events of October 18-20, 1995: interplanetary properties and as triggers for geomagnetic activity, J. Geophys. Res., 102, 14 049-14 064, 1997.

Liu, Y.: Halo coronal mass ejections and configuration of the ambient magnetic fields, Astrophys. J. Lett., 654, 171-174, 2007.

López Fuentes, M. C., Demoulin, P., Mandrini, C. H., and Van Driel-Gesztelyi, L.: The counterkink rotation of a non-Hale active region, Astrophys. J., 544, 540-549, 2000.

Lundquist, S.: Magnetostatic fields, Ark. Fys., 2361, 1950.

Lynch, B. J., Zurbuchen, T. H., Fisk, L. A., and Antiochos, S. K.: Internal structure of magnetic clouds: plasma and composition, J. Geophys. Res., 108, 1239-1253, 2003.

Mandrini, C. H., Pohjolainen, S., Dasso, S., Green, L. M., Démoulin, P., et al.: Interplanetary flux rope ejected from an Xray bright point, A \& A, 434, 725-740, 2005.

Marubashi, K.: Interplanetary magnetic flux ropes and solar filaments, in: Coronal Mass Ejections, Geophys. Monogr. Ser., 99, edited by: Crooker, N, Jocelyn, J., and Feynman, J., 147-156, AGU, Washington D.C., 1997.

Mulligan, T., Russell, C. T., and Luhmann, J. G.: Solar cycle evolution of the structure of magnetic clouds in the inner heliosphere, Geophys. Res. Lett., 25, 2959-2962, 1998.

Mulligan, T., Russell, C. T., and Gosling, J. T.: On interplanetary coronal mass ejection identification at $1 \mathrm{AU}$, in: Solar Wind Nine, edited by: Habbal, S. R., Esser, R., Hollweg, J. V., and Isenberg, P. A., AIP Conf. Proc. 471, 693, Woodbury, N.Y., 1999.

Neugebauer, M., Goldstein, R., and Goldstein, B. E.: Features observed in the trailing regions of interplanetary clouds from coronal mass ejections, J. Geophys. Res., 102, 19743-19751, 1997.

Pevtsov, A. A. and Balasubramaniam, K. S.: Helicity patterns on the Sun, Adv. Space Res., 32, 1867-1874, 2003.

Pick, M., Forbes, T. G., Mann, G., Cane, H. V., Chen, J., et al.: Multi-wavelength observations of CMEs and associated phenomena, Space Sci. Rev., 123, 341-382, 2006.

Qiu, J., Hu, Q., Howard, T. A., and Yurchyshyn, V. B.: On the magnetic flux budget in low-corona magnetic reconnection and interplanetary coronal mass ejection, Astrophys. J., 659, 758772, 2007

Reiner, M. J., Kaiser, M. L., and Bougeret, J.-L.: Coronal and interplanetary propagation of CME/shocks from radio, in situ and white-light observations, Astrophys. J., 663, 1369-1385, 2007.

Richardson, I. G. and Cane, H. V.: Regions of abnormally low proton temperature in the solar wind (1965-1991) and their association with ejecta, J. Geophys. Res., 100, 23 397-23 412, 1995.

Richardson, I. G., Cane, H. V., Lepri, S. T., Zurbuchen, T. H., and Gosling, J. T.: Spatial relationship of signatures of interplanetary coronal mass ejections, in: Solar Wind Ten, edited by: Velli, M., Bruno, R., and Malara, F., AIP Conf. Proc., 679, 681, Mellville, N.Y., 2003.
Rodriguez, L., Zhukov, A. N., Dasso, S., Mandrini, C. H., Cremades, H., et al.: Magnetic clouds seen at different locations in the heliosphere, Ann. Geophys., 26, 213-229, 2008, http://www.ann-geophys.net/26/213/2008/.

Scherrer, P. H., Bogart, R. S., Bush, R. I., Hoeksema, J. T., Kosovichev, A. G., Schou, J., et al.: The Solar Oscillations Investigation - Michelson Doppler Imager, Sol. Phys., 162, 129-188, 1995.

Schrijver, C. J., Title, A. M., Berger, T. E., Fletcher, L., et al.: A new view of the solar atmosphere by the Transition Region and Coronal Explorer, Sol. Phys., 187, 261-302, 1999.

Schwenn, R., Dal Lago, A., Huttunen, E., and Gonzalez, W. D.: The association of coronal mass ejections with their effects near the Earth, Ann. Geophys., 23, 1033-1059, 2005,

http://www.ann-geophys.net/23/1033/2005/.

Siscoe, G. L. and Suey, R. W.: Significance criteria for variance matrix applications (Significance criteria for comparing strength parameter against expectation from random group of vectors in variance matrix applications), J. Geophys. Res., 77, 1321-1322, 1972.

Smith, C. W., L'Heureux, J., Ness, N. F., Acuna, M. H., Burlaga, L. F., and Scheifele, J.: The ACE magnetic fields experiment, Space Sci. Rev., 86, 613, 1998.

Sonnerup, B. U. and Cahill, L. J.: Magnetopause structure and attitude from Explorer 12 observations, J. Geophys. Res., 72, 171183, 1967.

St. Cyr, O. C., Burkepile, J. T., Hundhausen, A. J., and Lecinski, A. R.: A comparison of ground-based and spacecraft observations of coronal mass ejections from 1980-1989, J. Geophys. Res., 104, 12 493-12 506, 1999.

St. Cyr, O. C., Howard, R. A., Sheeley, N. R., et al.: Properties of coronal mass ejections: SOHO LASCO observations from January 1996 to June 1998, J. Geophys. Res., 105, 18 169-18 185, 2000.

Sterling, A. C. and Hudson, H. S.: Yohkoh SXT observations of X-ray "dimming" associated with a halo coronal mass ejection, Astrophys. J., 491, L55-L58, 1997.

Sturrock, P. A.: Model of the high energy phase of solar flares, Nature, 211, 695-697, 1966.

Sturrock, P. A. and Smith, S. M.: Magnetic-field structure associated with coronal streamers, Sol. Phys., 5, 87-101, 1968.

Török, T. and Kliem, B.: Numerical simulations of fast and slow coronal mass ejections, Astronomische Nachrichten, 328, 743746, 2007.

Zhang, J., Dere, K. P., Howard, R. A., and Bothmer, V.: Identification of solar sources of major geomagnetic storms between 1996 and 2000, Astrophys. J., 582, 520-533, 2003.

Zwickl, R. D., Asbridge, J. R., Bame, S. J., et al.: Plasma properties of driver gas following interplanetary shocks observed by ISEE3, in: Solar Wind Five, edited by: Neugebauer, M., NASA Conf. Proc., 2280, 711, NASA, Washington D.C., 1983. 